\title{
Combinatorial properties of the noncommutative Faà di Bruno algebra
}

\author{
Jean-Paul Bultel
}

Received: 1 December 2011 / Accepted: 24 September 2012 / Published online: 13 October 2012

(C) Springer Science+Business Media New York 2012

\begin{abstract}
We give a new combinatorial interpretation of the noncommutative Lagrange inversion formula, more precisely, of the formula of Brouder-FrabettiKrattenthaler for the antipode of the noncommutative Faà di Bruno algebra.
\end{abstract}

Keywords Noncommutative symmetric functions · Combinatorial Hopf algebras · Lagrange inversion

\section{Introduction}

The classical Faà di Bruno algebra is the Hopf algebra of polynomial functions on the group of formal diffeomorphisms of the real line. The calculation of its antipode is equivalent to the Lagrange inversion formula, which gives the compositional inverse of any invertible formal power series in one variable with coefficients in a commutative algebra.

Formal power series in one variable with coefficients in a noncommutative algebra can be composed (by substitution of the variable), but this operation is not associative, so that they do not form a group. However, an analogue of the Faà di Bruno algebra still exists in this context. It is investigated in [1] in view of applications in quantum field theory, and in [1], one finds in particular a combinatorial formula for its antipode. This formula is rederived by Novelli and Thibon [10], who also show that it is equivalent to the noncommutative Lagrange formula of Gessel and Pak-PostnikovRetakh and can be obtained from it by a simple application of the antipode of the Hopf algebra of noncommutative symmetric functions.

\section{J.-P. Bultel (凶)}

Institut Gaspard Monge, Université Paris-Est Marne-la-Vallée, 5 Boulevard Descartes,

Champs-sur-Marne, 77454 Marne-la-Vallée cedex 2, France

e-mail: bultel@univ-mlv.fr 
Our main result is a new combinatorial interpretation of the formula of BrouderFrabetti-Krattenthaler. Namely, it is a combinatorial interpretation of the coefficients in the expansion of the antipode of the simple complete noncommutative symmetric functions in the basis of ribbons, $R_{I}$. We show that the coefficient of $R_{I}$ in this expansion is the number of nondecreasing parking functions smaller componentwise than some nondecreasing parking function $F$ obtained from $I$.

We also give an analogous combinatorial interpretation of the formula of Gessel and Pak-Postnikov-Retakh, and we explain how we can deduce these two interpretations from each other.

In [3], Foissy obtains, as a byproduct of his investigation of combinatorial Schwinger-Dyson equations, one-parameter families of Hopf algebras, interpolating respectively between symmetric functions and Faà di Bruno, and between noncommutative symmetric functions and the noncommutative Faà di Bruno algebra. In the last three sections, we are interested in the noncommutative family. We give in [2] a closed formula for the corresponding antipode, which is a natural deformation of the Brouder-Frabetti-Krattenthaler formula.

In [3], Foissy also shows that these Hopf algebras are generically isomorphic to the noncommutative Faà di Bruno algebra, except for some singular value, for which it is isomorphic to the Hopf algebra of noncommutative symmetric functions. In Sect. 9, we give a new description of the corresponding isomorphism. In Sect. 10, we define an algebra morphism which maps the antipode of the classical case to the one of the deformation. We give some properties of this morphism and describe its action on various bases. By means of these maps, we give an infinite family of functional equations of which the antipode of the classical case is a solution. In Sect. 11, we use this morphism to give another form of the deformed noncommutative Lagrange inversion formula, that is, a deformation of the formula of Gessel and Pak-Postnikov-Retakh. For these two deformed Lagrange inversion formulae, we also give combinatorial interpretations.

We follow the conventions of $[4,6]$. For the convenience of the reader, the most essential ones are recalled in Sect. 2.

\section{Conventions}

\subsection{Compositions}

Let $n$ be a nonnegative integer. A finite sequence $I=\left(i_{1}, \ldots, i_{s}\right)$ of positive integers is called a composition of $n$ if

$$
\sum_{k=0}^{s} i_{k}=n .
$$

We then write $I \vDash n$. The $i_{k}$ are the parts of $I,|I|=n$ is the weight of $I$, and the number $l(I)$ of parts in $I$ is the length of $I$. The multiplicity $m_{k}(I)$ of $k$ in $I$ is the number of parts in $I$ equal to $k$. We represent $I$ by a ribbon in which the lengths of the lines, read from the left to the right and from the bottom to the top, are the values 
of the parts of $I$. For example, (222) and (311) correspond respectively to

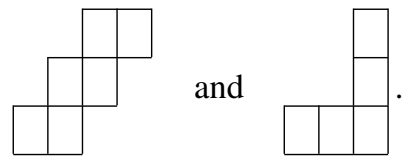

The conjugate composition of $I$ is the composition in which the parts are the lengths of the columns in the ribbon corresponding to $I$, read from the right to the left. For example, (1221) is the conjugate composition of (222), and (311) is the conjugate composition of itself. For any two compositions $I$ and $J$, we denote by $I \cdot J$ the composition obtained by concatenating $I$ and $J$, by $I^{\prime}$ the composition $\left(i_{l(I)}, \ldots, i_{1}\right)$, by $\tilde{I}$ the conjugate composition of $I$, and by $I^{\sharp}$ the conjugate composition of $I^{\prime}$. We use the notation $J \leq I$ to say that $J$ is a refinement of $I$, that is, for some $K \vDash l(J)$,

$$
\left\{\begin{array}{l}
j_{1}+j_{2}+\cdots+j_{k_{1}}=i_{1}, \\
j_{k_{1}+1}+\cdots+j_{k_{1}+k_{2}}=i_{2}, \\
\vdots
\end{array}\right.
$$

In such a case, we also say that $I$ is a reverse refinement of $J$.

\subsection{Noncommutative symmetric functions}

We denote by Sym the Hopf algebra of noncommutative symmetric functions. Its graded dual is the Hopf algebra QSym of quasi-symmetric functions. We denote by $A$ the underlying alphabet of the standard realization of noncommutative symmetric functions, and we identify any $F \in \mathbf{S y m}$ with its realization $F(A)$ when convenient. We denote by $S_{n}$ the $n$th complete noncommutative symmetric function and by $\Lambda_{n}$ the $n$th elementary noncommutative symmetric function. We set

$$
\sigma_{t}(A)=\sum_{n \geq 0} t^{n} S_{n}(A),
$$

and for any scalar $\alpha$, we define $S_{n}(\alpha A)$ as the coefficient of $t^{n}$ in

$$
\sigma_{t}(\alpha A)=\sigma_{t}(A)^{\alpha}=\left(\sum_{n \geq 0} t^{n} S_{n}(A)\right)^{\alpha} .
$$

This allows us to make sense of $F(\alpha A)$ when $F$ is any noncommutative symmetric function, since $\mathbf{S y m}$ is generated by the $S_{n}$.

We denote by $\left(R_{I}\right)$ the basis of ribbon noncommutative symmetric functions, and by $\left(S^{I}\right)$ and $\left(\Lambda^{I}\right)$ the multiplicative bases defined by

$$
S^{I}=S_{i_{1}} S_{i_{2}} \ldots S_{i_{l(I)}}
$$

and

$$
\Lambda^{I}=\Lambda_{i_{1}} \Lambda_{i_{2}} \ldots \Lambda_{i_{l(I)}} .
$$

These bases of Sym are parameterized by compositions of all integers. 


\subsection{Nondecreasing parking functions}

A nondecreasing parking function (also called a subexcedent function) of size $n$ is a nondecreasing sequence $F=\left(f_{1} \ldots f_{n}\right), f_{i} \leq i$ of $n$ nonnegative integers smaller than $(123 \ldots n)$ term by term. We denote by $\operatorname{NDPF}(n)$ the set of nondecreasing parking functions of size $n$. For a composition $I \vDash n$, we define $p(I)$ as the parking function $F$ of size $n$ such that $m_{k}(F)=i_{k}$ for all $k$. For example, $p(321)=(111223)$. For a nondecreasing parking function $F$ of size $n$, we $\operatorname{define} \operatorname{ev}(F)$ as the composition obtained by removing all the parts equal to zero in $\left(m_{1}(F), m_{2}(F), \ldots\right)$. For example, one has ev(1224) $=(121)$. As we can see, ev is surjective but not injective, and for all $I$, one has

$$
\operatorname{ev}(p(I))=I
$$

When $F=p(I)$ for some composition $I$, we say that $F$ is a packed nondecreasing parking function. Finally, we write $F \leq G$ if $f_{i} \leq g_{i}$ for all $i$, where $F$ and $G$ are two nondecreasing parking functions of the same size. For example, one has (112234) $\leq$ (122345).

\subsection{Cuts of a composition}

We shall need the following definitions.

Definition 2.1 Let $n>0$ be an integer, and $\phi$ a function from the set of the first $n$ nonzero integers to itself. We will say that $\phi$ is a cut function of size $n$ if

$$
\phi(1)=1
$$

and

$$
0 \leq \phi(k+1)-\phi(k) \leq 1
$$

for any $k$ such that this inequality makes sense.

Actually, cut functions of size $n$ coincide with packed nondecreasing parking functions of size $n$. Namely, each cut function $\phi$ can be identified with the parking function

$$
(\phi(1), \phi(2), \ldots, \phi(n)) .
$$

We identify any cut function with its corresponding packed nondecreasing parking function when convenient.

Definition 2.2 For all compositions $J$ and for all cut functions $\phi$ of size $l(J)$, let us define the cut of $J$ corresponding to $\phi$ as the following sequence of compositions:

$$
D_{J, \phi}=\left(J^{(1)}, J^{(2)}, \ldots\right),
$$

where for all $n, J^{(n)}$ is defined by

$$
J^{(n)}=\left(j_{k}, j_{k+1}, j_{k+2}, \ldots, j_{s}\right),
$$


where $k$ and $s$ are such that $\phi(x)=n$ for all integers $x \in[k, s]$, and $\phi(x) \neq n$ for all other values of $x$, so that

$$
\phi(k)=\phi(k+1)=\cdots=\phi(s)=n .
$$

We also define the length of a cut as the number of compositions in the cut.

Example 2.3 Consider the composition $J=(13122)$ and the cut function $\phi=$ (11233) of size $l(J)$. Then,

$$
D_{J, \phi}=((13),(1),(22)),
$$

and the length of this cut is 3 .

Definition 2.4 Let $I$ be a composition and $J$ a refinement of $I$. We then define the $I$-cut of $J$ as the cut

$$
\left(J^{(1)}, J^{(2)}, \ldots\right)
$$

such that for all $k, J^{(k)} \vDash i_{k}$. We also define $\phi_{I, J}$ as the corresponding cut function. Note that it is such that for all $s$,

$$
\sum_{\phi_{I, J}(k)=s} j_{k}=i_{s}
$$

Example 2.5 Consider the composition $I=(323)$. Then, $J=(212111)$ is a refinement of $I$, and the $I$-cut of $J$ is

$$
((21),(2),(111))
$$

so that

$$
\phi_{I, J}=(112333) .
$$

Definition 2.6 Let $I$ and $J$ be two compositions of same weight $n$ such that

$$
J \leq I
$$

We will say that a cut $D_{\psi, J}$ is $I$-admissible and that the corresponding cut function $\psi$ is $(I, J)$-admissible if

$$
\psi(2)=2
$$

and if for all $k$ and $s$ such that $\phi_{I, J}(k)=\phi_{I, J}(s)$,

$$
\psi(k) \neq \psi(s) .
$$

For example, if $J \neq I$, the $I$-cut of $J$ is not $I$-admissible 
Example 2.7 Set $I=(323)$ and $J=(212111)$ again. Then, the cut

$$
((2),(121),(1),(1))
$$

of $J$ is $I$-admissible, because the corresponding cut function (122234) is $(I, J)$ admissible. The cut

$$
((2),(12),(1),(1),(1))
$$

is also $I$-admissible, because the corresponding cut function (122345) is also $(I, J)$ admissible. However, the cut

$$
((2),(12),(11),(1))
$$

is not $I$-admissible. Indeed, the corresponding cut function is $\psi=(122334)$, and one has $\phi_{I, J}=(112333)$, so that $\psi(4)=\psi(5)$ and $\phi_{I, J}(4)=\phi_{I, J}(5)$.

\section{The Hopf algebra $\mathcal{H}$ of noncommutative formal diffeomorphisms}

\subsection{The noncommutative Lagrange inversion formula}

The classical Lagrange inversion formula for the reversion of formal power series can be interpreted in terms of classical symmetric functions (see [9], Ex. 24, p. 35, Ex. 25 , p. 132, [7], Sect. 2.4, and [8]). Similarly, for various noncommutative analogues of the Lagrange inversion formula (see [1, 5] and [11]), Novelli and Thibon give in [10] interpretations in terms of noncommutative symmetric functions.

Brouder, Frabetti, and Krattenthaler obtain in [1] a form of the noncommutative Lagrange inversion formula, that is, an explicit formula for the antipode of the Hopf algebra $\mathcal{H}$ of noncommutative formal diffeomorphisms, also known as the noncommutative Faà di Bruno algebra. The elements of $\mathcal{H}$ can be identified with noncommutative symmetric functions by means of the correspondence $a_{n}$ (of formula (2.16) in [1]) $=S_{n}$ (of $\mathbf{S y m}$ ), which identifies $\mathcal{H}$ with $\mathbf{S y m}$ as an associative algebra. Under this correspondence, the coproduct $\Delta$ on $\mathcal{H}$ takes the form

$$
\Delta S_{n}(A)=\sum_{k=0}^{n} S_{k}(A) \otimes S_{n-k}((k+1) A) .
$$

We denote by $s: F \mapsto F^{\star}$ the antipode of the Hopf algebra $\mathcal{H}$. The value of the coefficient $\alpha_{I}$ defined by

$$
S_{n}^{\star}=\sum_{I \models n} \alpha_{I} S^{I}
$$

is given in [1] as

$$
\alpha_{I}=(-1)^{l(I)} \sum_{\left(a_{1}, \ldots, a_{l(I)-1}\right)} \prod_{k=1}^{l(I)-1}\left(\begin{array}{c}
i_{k}+1 \\
a_{k}
\end{array}\right)
$$


In formula (28), the sum is taken over the set $\mathcal{A}_{l(I)-1}$ of all the sequences $\left(a_{1}, \ldots, a_{l(I)-1}\right)$ where the $a_{i}$ are positive integers such that

$$
a_{1}+\cdots+a_{l(I)-1}=l(I)-1
$$

and for all $1 \leq j<l(I)-1$,

$$
a_{1}+\cdots+a_{j} \leq j
$$

Note that the set $\mathcal{A}_{n}$ is in bijection with the Catalan set of nondecreasing parking functions of length $n$. An explicit bijection can be obtained by associating $\left(\rho_{1}, \rho_{2}, \ldots\right) \in \mathcal{A}_{n}$ with the parking function

$$
(\underbrace{1, \ldots, 1}_{\rho_{1} \text { times }}, \underbrace{2, \ldots, 2}_{\rho_{2} \text { times }}, \ldots)
$$

This remark allows us to rewrite (28) as

$$
\alpha_{I}=(-1)^{l(I)} \sum_{F \in \operatorname{NDPF}(l(I)-1)} \prod_{k=1}^{l(I)-1}\left(\begin{array}{c}
i_{k}+1 \\
m_{k}(F)
\end{array}\right) .
$$

Gessel [5] and Pak-Postnikov-Retakh [11] give another version of the noncommutative Lagrange inversion formula. Novelli and Thibon [10] show that this formula can also be interpreted in terms of noncommutative symmetric functions. They also show that it is equivalent to the Brouder-Frabetti-Krattenthaler formula. Namely, it corresponds to the evaluation of the coefficient $\hat{\alpha}_{I}$ in

$$
S_{n}^{\star}(-A)=\sum_{I \models n} \hat{\alpha}_{I} S^{I}(A),
$$

whose value is given by

$$
\hat{\alpha}_{I}=\sum_{\left(a_{1}, \ldots, a_{l(I)-1}\right)} \prod_{k=1}^{l(I)-1}\left(\begin{array}{c}
i_{k} \\
a_{k}
\end{array}\right)
$$

where the parameters of the sum are the same as in (28). Equivalently,

$$
\hat{\alpha}_{I}=\sum_{F \in \operatorname{NDPF}(l(I)-1)} \prod_{k=1}^{l(I)-1}\left(\begin{array}{c}
i_{k} \\
m_{k}(F)
\end{array}\right) .
$$

In [2], we give a recurrence formula for the coefficient $\beta_{I}$ in

$$
S_{n}^{\star}=\sum_{I \models n} \beta_{I} R_{I}
$$


that is,

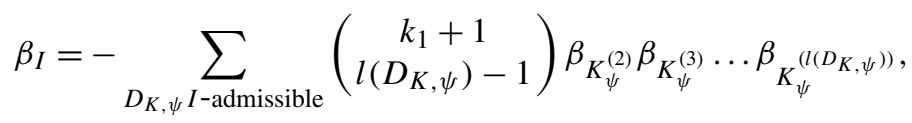

where the sum is taken over all the $I$-admissible cuts of all the refinements of $I$. In the sequel, we denote respectively by $\alpha_{I}, \beta_{I}, \delta_{I}$ and $\hat{\alpha}_{I}, \hat{\beta}_{I}, \hat{\delta}_{I}$ the coefficients in

$$
S_{n}^{\star}=\sum_{I \models n} \alpha_{I} S^{I}=\sum_{I \models n} \beta_{I} R_{I}=\sum_{I \models n} \delta_{I} \Lambda^{I}
$$

and

$$
S_{n}^{\star}(-A)=\sum_{I \models n} \hat{\alpha}_{I} S^{I}(A)=\sum_{I \models n} \hat{\beta}_{I} R_{I}(A)=\sum_{I=n} \hat{\delta}_{I} \Lambda^{I}(A) .
$$

For any sequence $\left(c_{i}\right)$ of coefficients and any composition $I$, we set

$$
c^{I}=\prod_{k=1}^{l(I)} c_{i_{k}}
$$

\subsection{An involution}

One has

$$
\begin{aligned}
\Delta\left(\sigma_{1}(A)\right) & =\sum_{n \geq 0} \sum_{k=0}^{n} S_{k}(A) \otimes S_{n-k}((k+1) A) \\
& =\sum_{k \geq 0} S_{k}(A) \otimes \sigma_{1}((k+1) A) \\
& =\sum_{k \geq 0} S_{k} \otimes \sigma_{1}(A)^{k+1},
\end{aligned}
$$

that is,

$$
1=\sum_{k \geq 0} S_{k} \sigma_{1}^{\star}(A)^{k+1} .
$$

Then, $\sigma_{1}^{\star}$ is the same as the series $h$ in formula (47) in [10], and formula (50) in [10] allows us to define the series $g$ of [10] as

$$
g(A)=\sigma_{1}^{\star}(-A)=\sum_{n \geq 0} S_{n}^{\star}(-A)
$$

Novelli and Thibon show in [10] that the series $g$ is invariant under the involution

$$
v: S^{I} \mapsto S^{\tilde{I}}
$$


Since one has $S^{I}(-A)=(-1)^{|I|} \Lambda^{I}(A)$ for all compositions $I$, one obtains

$$
g(-A)=\sum_{I}(-1)^{|I|} \hat{\alpha}_{I} \Lambda^{I}=\sum_{I}(-1)^{|I|} \hat{\alpha}_{I} \Lambda^{\tilde{I}} .
$$

From that we deduce the following proposition.

Proposition 3.1 The $\delta_{I}$ satisfy the relation

$$
\delta_{I}=\delta_{\tilde{I}}
$$

Novelli and Thibon also show in [10] that $v$ maps $R_{I}$ to $(-1)^{l(I)-1} \Lambda^{\tilde{I}}$, so that

$$
g=\sum_{I} \hat{\beta}_{I} R_{I}=\sum_{I}(-1)^{l(I)+1} \hat{\beta}_{I} \Lambda^{\tilde{I}}
$$

Hence,

$$
\sigma_{1}^{\star}(A)=\sum_{I} \hat{\beta}_{I} R_{I}(-A)=\sum_{I}(-1)^{|I|+l(I)+1} \hat{\beta}_{I} S^{\tilde{I}}
$$

We shall need the expansion of the $\Lambda^{I}$ in the basis $\left(R_{J}\right)$, and the one of the $R_{I}$ in the basis $\left(\Lambda^{J}\right)$. When $I$ is a composition with two parts, one has

$$
\Lambda^{I}=R_{1^{i_{1}}} R_{1^{i_{2}}}=R_{1^{i_{1}+i_{2}}}+R_{1^{i_{1}-1}, 2,1^{i_{2}-1}} .
$$

By induction, one has in the general case

$$
\Lambda^{I}=\sum_{J \leq I^{\sharp}} R_{J}
$$

Denote by $\left(\Xi_{I}\right)$ the basis defined by $\Xi_{I}=\Lambda^{I^{\sharp}}$. We then obtain

$$
\Xi_{I}=\sum_{J \leq I} R_{J}
$$

Hence,

$$
R_{I}=\sum_{J \leq I}(-1)^{l(I)+l(J)} \Xi_{J}=\sum_{J \geq I^{\sharp}}(-1)^{l\left(J^{\sharp}\right)+l(I)} \Lambda^{J} .
$$

Note that $l(J)+l\left(J^{\sharp}\right)=|J|+1=|I|+1$, so that (52) can be rewritten as

$$
R_{I}=\sum_{J \geq I^{\sharp}}(-1)^{|I|+1+l(I)+l(J)} \Lambda^{J} .
$$

Hence,

$$
R_{I}(-A)=\sum_{J \geq I^{\sharp}}(-1)^{l(J)+l(I)+1} S^{J} .
$$


On the other hand,

$$
S^{I}=\sum_{J \geq I} R_{J}
$$

so that (48) can be rewritten as

$$
\sigma_{1}^{\star}(A)=\sum_{I} \hat{\beta}_{I} \sum_{J \geq I^{\sharp}}(-1)^{l(J)+l(I)+1} S^{J}=\sum_{I}(-1)^{|I|+l(I)+1} \hat{\beta}_{I} \sum_{J \geq \tilde{I}} R_{J},
$$

so that

$$
\sigma_{1}^{\star}(A)=\sum_{J}\left(\sum_{I \geq J^{\sharp}}(-1)^{l(J)+l(I)+1} \hat{\beta}_{I}\right) S^{J}=\sum_{J}\left(\sum_{I \geq \tilde{J}}(-1)^{|I|+l(I)+1} \hat{\beta}_{I}\right) R_{J} .
$$

Finally, we then have

$$
\begin{aligned}
\sigma_{1}^{\star}(A) & =\sum_{J}(-1)^{l(J)}\left(\sum_{I \geq \tilde{J}}(-1)^{l(I)+1} \hat{\beta}_{I}\right) S^{J^{\prime}} \\
& =\sum_{J}(-1)^{|J|}\left(\sum_{I \geq \tilde{J}}(-1)^{l(I)+1} \hat{\beta}_{I}\right) R_{J} .
\end{aligned}
$$

From that we deduce the following proposition.

Proposition 3.2 The expansion of $\sigma_{1}^{\star}$ in the basis $\left(R_{I}\right)$ is linked to its expansion in the basis $S^{I}$ by

$$
\beta_{I}=(-1)^{|I|-l(I)} \alpha_{I^{\prime}}
$$

Remark 1 Since the sign of $\alpha_{I}$ is $(-1)^{l(I)}$, we can deduce from this proposition that the sign of $\beta_{I}$ is $(-1)^{|I|}$. Furthermore, since $\alpha_{I}$ does not depend on the last part of $I$, we deduce that $\left|\beta_{I}\right|$ does not depend on the first part of $I$. More precisely,

$$
\beta_{n \cdot I}=(-1)^{n+1} \beta_{1 \cdot I}
$$

Proposition 3.2 allows us to say that the Brouder-Frabetti-Krattenthaler formula is equivalent to the evaluation of the coefficients $\beta_{I}$. On the other hand, (45) allows us to say that the formula of Gessel and Pak-Postnikov-Retakh is equivalent to the evaluation of the coefficients $\delta_{I}$. The following two sections are devoted to giving recurrence formulae for these two families of coefficients, in order to give combinatorial interpretations for them.

\section{A recurrence formula for the $\beta_{I}$}

This section is devoted to the proof of the following theorem. 
Theorem 4.1 The $\beta_{I}$ are determined by induction by

$$
\beta_{n}=(-1)^{n}
$$

when $I=(n)$ and by

$$
\beta_{I}=\beta_{i_{1}, i_{2}, \ldots, i_{s-1}+1, i_{s}-1}+\beta_{i_{1}, i_{2}, \ldots, i_{s-1}} \beta_{i_{s}}
$$

when $l(I)>1$.

The case where $I$ has at most two parts can be immediately deduced from (28) and (59). When $I$ is a composition with two parts, we obtain

$$
\beta_{I}=(-1)^{i_{2}+i_{1}}\left(\begin{array}{c}
i_{2}+1 \\
1
\end{array}\right)
$$

so that one has $\beta_{I}=(-1)^{i_{1}+i_{2}}\left(i_{2}+1\right)$, and $\beta_{I}$ verifies the theorem. Now, we shall need the following definition.

Definition 4.2 Let $I$ be a composition of weight $n$, and let $k$ be a nonnegative integer such that $k \leq n$. Denote by $s$ the integer such that

$$
i_{1}+\cdots+i_{s} \leq k \leq i_{1}+\cdots+i_{s}+i_{s+1} .
$$

Then, we define $I_{g}(k)$ as the composition obtained by removing the parts equal to zero in

$$
\left(i_{1}, i_{2}, \ldots, i_{s}, k-i_{1}-i_{2}-\cdots-i_{s}\right) .
$$

We also define $I_{d}(k)$ as the one obtained by removing the parts equal to zero in

$$
\left(i_{1}+\cdots+i_{s}+i_{s+1}-k, i_{s+1}, i_{s+2}, \ldots\right) \text {. }
$$

Note that $I_{g}(0)=I_{d}(n)=\emptyset$ and $I_{g}(n)=I_{d}(0)=I$. For example, for $I=(2,3,4)$, one has

$$
I_{g}(3)=(2,1), \quad I_{d}(7)=(2), \quad I_{g}(8)=(2,3,3), \quad I_{d}(3)=(2,4) .
$$

\subsection{A recurrence formula for $\beta_{I}$}

Now, suppose that $I$ has at least three parts. Denoting by $\hat{I}$ the composition obtained by replacing the first part of $I$ by 1 , Remark 1 allows us to rewrite (37) as

$$
\beta_{I}=(-1)^{i_{1}} \sum_{D_{K, \psi} \hat{I} \text {-admissible }}\left(\begin{array}{c}
2 \\
l\left(D_{K, \psi}\right)-1
\end{array}\right) \beta_{K_{\psi}^{(2)}} \beta_{\left.\left.K_{\psi}^{(3)} \ldots \beta_{K_{\psi}\left(l \left(D_{K}, \psi\right.\right.}\right)\right)}
$$

Indeed, since the $K$ are refinements of $\hat{I}$ in this formula, one has $k_{1}=1$, so that $k_{1}+1=2$. Hence, the binomial coefficient is equal to 0 for the cuts with length 
greater than 4. This remark allows us to consider only the cuts of length smaller than 3. Furthermore, we know from (21) that the first part of these cuts is $\left(k_{1}\right)=$ $\left(\hat{i}_{1}\right)=(1)$. The other one or two parts must then form a refinement of $\left(i_{2}, i_{3}, \ldots\right)$.

Now, this restriction on the number of parts allows us to say from (22) that this refinement must be obtained by splitting at most one part of $\left(i_{2}, i_{3}, \ldots\right)$. The only possibility to form an $I$-admissible cut by splitting a part is to make one part with all that is at the left of where we have split, and another one with all that is at the right of it. Note that if one does not halve any part of the composition $\left(i_{2}, i_{3}, \ldots\right)$, then any considered cut will be $I$-admissible.

Denoting by $\bar{I}=\left(i_{2}, \ldots, i_{l(I)}\right)$, we can then rewrite (68) as

$$
\beta_{I}=(-1)^{i_{1}}\left(\left(\begin{array}{l}
2 \\
1
\end{array}\right) \beta_{\bar{I}}+\left(\begin{array}{l}
2 \\
2
\end{array}\right) \sum_{k=1}^{|\bar{I}|-1} \beta_{\bar{I}_{g}(k)} \beta_{\bar{I}_{d}(k)}\right),
$$

that is,

$$
\beta_{I}=(-1)^{i_{1}} \sum_{k=0}^{|\bar{I}|} \beta_{\bar{I}_{g}(k)} \beta_{\bar{I}_{d}(k)} .
$$

For instance, let us evaluate $\beta_{I}$ for $I=(2,4,3)$. The following figure is a graphical representation of $I$, in which the sizes of the rectangles are the values of the parts:

$$
I=\begin{array}{|l|l|l|}
\hline & & \\
\hline
\end{array}
$$

Let us consider any refinement of $I$, for instance, $J=(2,1,3,2,1)$. Then, the $I$-cut of $J$ corresponds to the cut function $\phi$ such that

$$
\phi(1)=1, \quad \phi(2)=2, \quad \phi(3)=2, \quad \phi(4)=3, \quad \phi(5)=3 .
$$

The $I$-cut of $J$ can be represented as

\begin{tabular}{|l||l|l||l|l|}
\hline 1 & 2 & 2 & 3 & 3 \\
\hline
\end{tabular}

Let us try to make an $I$-admissible cut of $J$, and denote by $\psi$ the corresponding cut function. From the condition $\psi(2)=2$, the chosen refinement must satisfy $i_{1}=j_{1}$, which is true for our $J$. From the other condition we must have $\psi(i) \neq \psi(j)$ when $\phi(i)=\phi(j)$. Then, the smaller $I$-admissible cut of $J$ that we can obtain is

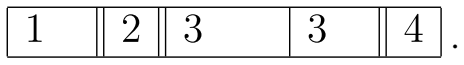

The length of this cut is 4 , and we can see that the only possibilities to build a cut of size smaller than 3 are the following three ones. One can obtain $J$ by splitting one part of $I$ (this part have to be at least the second), and change the value of $\psi$ at the corresponding place, as in this figure

\begin{tabular}{|l||l||l|l|}
\hline 1 & 2 & 3 & 3 \\
\hline
\end{tabular}


Another possibility is to consider that $I=J$ and to cut a second time anywhere at the right of the first part (in our example, we have no choice since $l(I)=3$ ):

\begin{tabular}{|l||l||l|}
\hline 1 & 2 & 3 \\
\hline
\end{tabular}

The last possibility is to consider that $I=J$ and to change the value of $\psi$ only between the first part and the second one. In such a case, the value of $\psi$ is 2 everywhere at the right. Then, we obtain a cut with two parts.

The first two constructions give a contribution to the term of $\left(\begin{array}{l}2 \\ 2\end{array}\right)$ in (69). The third one gives a contribution to the term of $\left(\begin{array}{l}2 \\ 1\end{array}\right)$, and we then obtain the specialization of formula (70) corresponding to our example. Namely, we obtain

$$
\beta_{(243)}=2 \beta_{(43)}+\beta_{1} \beta_{(33)}+\beta_{2} \beta_{(23)}+\beta_{3} \beta_{(13)}+\beta_{4} \beta_{3}+\beta_{(41)} \beta_{2}+\beta_{(42)} \beta_{1} \text {. }
$$

\subsection{Proof of the theorem}

Let $I$ be a composition with at least three parts. We are going to derive Theorem 4.1 by induction on $|I|$. In order to do that, we suppose that (62) is true for all the compositions $K$ such that $|K|<|I|$. We denote by $s$ and $n$ the last two parts of $I$ in this order, so that $n$ is the last one. Let $J$ be the composition obtained from $I$ by removing its first part and its last two parts. Set also $D=\beta_{I}-\beta_{\left(i_{1}\right) \cdot J \cdot(s+1, n-1)}$, and define $A$ and $B$ by

$$
\begin{aligned}
A= & \sum_{k=0}^{|J|}\left(\beta_{J_{g}(k)} \beta_{J_{d}(k) \cdot(s, n)}-\beta_{J_{g}(k)} \beta_{J_{d}(k) \cdot(s+1, n-1)}\right) \\
& +\sum_{k=1}^{s-1}\left(\beta_{J \cdot(k)} \beta_{(s-k, n)}-\beta_{J \cdot(k)} \beta_{(s-k+1, n-1)}\right)
\end{aligned}
$$

and

$$
\begin{aligned}
B= & \beta_{J \cdot(s)} \beta_{n}-\beta_{J \cdot(s)} \beta_{(1, n-1)} \\
& +\sum_{k=1}^{n}\left(\beta_{J \cdot(s, k)} \beta_{n-k}-\beta_{J \cdot(s+1, k-1)} \beta_{n-k}\right) .
\end{aligned}
$$

We deduce from (70) that

$$
(-1)^{i_{1}} D=A+B .
$$

Furthermore, by setting $\hat{J}=J \cdot(s)$ and applying Theorem 4.1 to compositions $K$ such that $|K|<|I|$, we obtain

$$
A=\sum_{k=0}^{|\hat{J}|-1} \beta_{\hat{J}_{g}(k)} \beta_{\hat{J}_{d}(k)} \beta_{n} .
$$


Then, we deduce from (70) that

$$
A+\beta_{\hat{J}} \beta_{n}=(-1)^{i_{1}} \beta_{\left(i_{1}\right) \cdot \hat{J}} \beta_{n} .
$$

Set $C=B-\beta_{\hat{J}} \beta_{n}$. From (78), we only have to show that $C=0$ to finish the proof of the theorem.

We have $\hat{J}=J \cdot(s)$. From the expansion of $B$ we can then see that the term $\beta_{\hat{J}} \beta_{n}$ appears two times with opposite signs in the expansion of $C$. Hence,

$$
C=-\beta_{\hat{J}} \beta_{(1, n-1)}+\sum_{k=1}^{n}\left(\beta_{\hat{J} \cdot(k)} \beta_{n-k}-\beta_{J \cdot(s+1, k-1)} \beta_{n-k}\right) .
$$

Now, for $1 \leq k \leq n$, since one has $|\hat{J} \cdot k|<|I|$, the application of Theorem 4.1 to $\hat{J} \cdot k$ leads to

$$
\beta_{\hat{J} \cdot(k)}-\beta_{J \cdot(s+1, k-1)}=\beta_{\hat{J}} \beta_{k} .
$$

By multiplying both sides of this equality by $\beta_{n-k}$, one can rewrite (81) as

$$
C=-\beta_{\hat{J}} \beta_{(1, n-1)}+\sum_{k=1}^{n} \beta_{\hat{J}} \beta_{k} \beta_{n-k}
$$

Now, we have $|(1, n-1)|<|I|$, so that we can apply the theorem to the composition $(1, n-1)$. Iteratively we obtain

$$
\beta_{(1, n-1)}=\beta_{(2, n-2)}+\beta_{1} \beta_{n-1}=\beta_{(3, n-3)}+\beta_{2} \beta_{n-2}+\beta_{1} \beta_{n-1}=\cdots,
$$

and the last step is

$$
\beta_{(1, n-1)}=\sum_{k=1}^{n} \beta_{k} \beta_{n-k} .
$$

From (83) we deduce that $C=0$, and we obtain our result.

\section{A recurrence formula for the $\delta_{I}$}

5.1 Some properties of the $\delta_{I}$

Let $I$ and $J$ be two compositions with the same length. Since

$$
l\left(J^{\sharp}\right)+l(I)=l(J)+l(I)+|I|+1=l\left(I^{\sharp}\right)+l(J),
$$

one can rewrite (52) as

$$
R_{I}=\sum_{J \geq I^{\sharp}}(-1)^{l(J)+l\left(I^{\sharp}\right)} \Lambda^{J} .
$$


Hence,

$$
\delta_{I}=\sum_{I \geq J^{\sharp}}(-1)^{l(J)+l\left(I^{\sharp}\right)} \beta_{J}=\sum_{J \geq I^{\sharp}}(-1)^{l(J)+l\left(I^{\sharp}\right)} \beta_{J} .
$$

Now, for all compositions $I$, we have the identity $\delta_{I}=\delta_{\tilde{I}}$ (46). Hence,

$$
\delta_{I}=\sum_{J \geq I^{\prime}}(-1)^{l(I)+l(J)} \beta_{J} .
$$

From this formula and from Theorem 4.1 we deduce the value of $\delta_{n}$. Note that since $(\tilde{n})=\left(1^{n}\right)$, we can also deduce the value of $\delta_{1^{n}}$, so that we can give the following proposition.

Proposition 5.1 The coefficients $\delta_{n}$ and $\delta_{1^{n}}$ are given by

$$
\delta_{n}=\delta_{1^{n}}=(-1)^{n} .
$$

From (89) and (59) we can also deduce the following proposition.

Proposition 5.2 The value of $\left|\delta_{I}\right|$ does not depend on the last part of I. Furthermore, this value does not change by adding a part 1 at the beginning of I. More precisely, one has for all I and $n$,

$$
\delta_{I \cdot(n)}=(-1)^{n-1} \delta_{I \cdot(1)}
$$

and

$$
\delta_{\left(1^{n}\right) \cdot I}=(-1)^{n} \delta_{I}
$$

These two equalities can be deduced from each other by using $\delta_{I}=\delta_{\tilde{I}}$. A numerical example of how to obtain (91) is

$$
\delta_{(23)}=\beta_{(32)}-\beta_{5}=-\left(\beta_{(42)}-\beta_{6}\right)=-\delta_{(24)} .
$$

\subsection{A recurrence formula for $\delta_{I}$}

We give the following theorem.

Theorem 5.3 For any composition $J, \delta_{J}$ is given by Propositions 5.1 and 5.2 and the following recurrence formula:

$$
\delta_{(n) \cdot(k) \cdot I}=-\left(\delta_{(n-1) \cdot k \cdot I}+\delta_{(n+k-1) \cdot I}\right),
$$

where $n>1, k>0$, and $l(I) \geq 0$.

By applying (46) to this theorem, one can immediately deduce the following: 
Corollary 5.4 For any composition I, any $k>1$, and any $n>0$, one has

$$
\delta_{I \cdot(k) \cdot\left(1^{n}\right)}=-\left(\delta_{I \cdot(k) \cdot\left(1^{n-1}\right)}+\delta_{I \cdot(k-1) \cdot\left(1^{n}\right)}\right) .
$$

This formula, together with Propositions 5.1 and 5.2, also determines all the $\delta_{I}$.

The rest of this section is devoted to the proof of Theorem 5.3. We shall need the following lemma.

Lemma 5.5 Let I be a composition of length $s>1$. Then,

$$
\beta_{I}-\beta_{\left(i_{1}, \ldots, i_{s-2}, i_{s-1}+i_{s}\right)}+\beta_{\left(i_{1}, \ldots, i_{s-1}, i_{s}-1\right)}=0 .
$$

Proof By applying some iterations of (62) as in (84), we obtain from $\beta_{n}=(-1)^{n}$ that

$$
\beta_{I}=\sum_{k=0}^{i_{s}}(-1)^{i_{s}-k} \beta_{\left(i_{1}, \ldots, i_{s-2}, i_{s-1}+k\right)} .
$$

By expanding $\beta_{I}$ and $\beta_{\left(i_{1}, \ldots, i_{s-1}, i_{s}-1\right)}$ as in this formula at the left of the equality in (96), one obtains a sum equal to zero.

Example 5.6 By applying (97), one obtains for $I=(2,2,2)$,

$$
\beta_{(222)}-\beta_{(24)}+\beta_{(221)}=\left(\beta_{(24)}-\beta_{(23)}+\beta_{(22)}\right)-\beta_{(24)}+\left(\beta_{(23)}-\beta_{(22)}\right)=0 .
$$

We are now able to give a proof of Theorem 5.3. Let $I$ be a composition as in the theorem, and denote by $J$ the composition obtained by subtracting 1 from the first part of $I$. Then, there are two types of reverse refinements of $J$. The first ones are obtained by concatenating $\left(j_{1}\right)$ and a reverse refinement of $\bar{I}=\left(j_{2}, j_{3}, \ldots\right)$. The second ones are the reverse refinements of $K=\left(j_{1}+j_{2}, j_{3}, \ldots\right)$.

Since

$$
\delta_{J}+\delta_{K}=\sum_{L \geq J}(-1)^{l(J)+l(L)} \beta_{L}+\sum_{L \geq K}(-1)^{l(L)+l(K)} \beta_{K},
$$

we then have

$$
\begin{aligned}
\delta_{J}+\delta_{K}= & \sum_{L \geq K}(-1)^{l(J)+l(L)} \beta_{L}+\sum_{L \geq \bar{I}}(-1)^{l(J)+l(L)+1} \beta_{\left(j_{1}\right) \cdot L} \\
& +\sum_{L \geq K}(-1)^{l(K)+l(L)} \beta_{L} .
\end{aligned}
$$

Now, $l(J)=1+l(K)$, so that

$$
\delta_{J}+\delta_{K}=\sum_{L \geq \bar{I}}(-1)^{l(J)+l(L)+1} \beta_{\left(j_{1}\right) \cdot L} .
$$


Hence,

$$
\delta_{I}+\delta_{J}+\delta_{K}=\sum_{L \geq I}(-1)^{l(I)+l(L)} \beta_{L}+\sum_{L \geq \bar{I}}(-1)^{l(J)+l(L)+1} \beta_{\left(j_{1}\right) \cdot L},
$$

that is, by setting $M=\left(i_{1}+i_{2}, i_{3}, \ldots\right)$,

$$
\begin{aligned}
\delta_{I}+\delta_{J}+\delta_{K}= & \sum_{L \geq \bar{I}}(-1)^{l(I)+l(L)+1} \beta_{\left(i_{1}\right) \cdot L} \\
& +\sum_{L \geq M}(-1)^{l(I)+l(L)} \beta_{L}+\sum_{L \geq \bar{I}}(-1)^{l(J)+l(L)+1} \beta_{\left(j_{1}\right) \cdot L} .
\end{aligned}
$$

On the other hand, one has $j_{1}=i_{1}-1, l(I)=l(J)$, and $l(M)=l(I)-1$. Furthermore, the refinements of $M$ are the compositions obtained by adding $i_{1}$ to the first part of refinements of $\bar{I}$. Hence,

$$
\begin{aligned}
\delta_{I}+\delta_{J}+\delta_{K}= & \sum_{L \geq \bar{I}}(-1)^{l(I)+l(L)+1} \beta_{\left(i_{1}\right) \cdot L} \\
& +\sum_{L \geq \bar{I}}(-1)^{l(I)+l(L)} \beta_{\left(i_{1}+l_{1}, l_{2}, \ldots\right)}+\sum_{L \geq \bar{I}}(-1)^{l(I)+l(L)+1} \beta_{\left(i_{1}-1\right) \cdot L},
\end{aligned}
$$

that is,

$$
\delta_{I}+\delta_{J}+\delta_{K}=\sum_{L \geq \bar{I}}(-1)^{l(I)+l(L)+1}\left(\beta_{\left(i_{1}\right) \cdot L}-\beta_{\left(i_{1}+l_{1}, l_{2}, \ldots\right)}+\beta_{\left(i_{1}-1\right) \cdot L}\right) .
$$

This sum is equal to zero by Lemma 5.5. From that we deduce our result.

\section{Combinatorial interpretations}

6.1 A combinatorial interpretation of the $\beta_{I}$

We shall need the following definition.

Definition 6.1 Let $I$ be a composition of length $s$. Then, we will say that a finite sequence $\phi=\left(\phi_{1}, \phi_{2}, \ldots, \phi_{s-1}\right)$ of integers of length $s-1$ such that

$$
\phi_{1} \leq i_{s}
$$

is a flow from right to left in $I$ if for all $1 \leq k \leq s-2$,

$$
\phi_{k+1} \leq i_{s-k}+\phi_{k} .
$$


We denote by $\Phi_{I}$ the set of these sequences, and for $\phi \in \Phi_{I}$, we denote by $\phi(I)$ the composition obtained by removing the parts equal to 0 in

$$
\left(i_{1}+\phi_{s-1}, i_{2}+\phi_{s-2}-\phi_{s-1}, \ldots, i_{s-1}+\phi_{1}-\phi_{2}, i_{s}-\phi_{1}\right) .
$$

Note that one cannot obtain negative parts in (108). Indeed, one has $i_{1}+\phi_{s-1} \geq 0$ and $i_{s}-\phi_{1} \geq 0$ (106). The other parts of $\phi(I)$ in (108) are of type $i_{s-k}+\phi_{k}+\phi_{k+1}$ with $1 \leq k \leq s-2$, so that they are also nonnegative (107).

Definition 6.1 will permit us to give a combinatorial interpretation for (62). After some iterations of (62), we obtain, for any composition $|I|$ with $|I| \geq 0$ and any two integers $k$ and $n$,

$$
\beta_{I \cdot(k) \cdot(n)}=\sum_{s=0}^{n} \beta_{I \cdot(k+s)} \beta_{n-s} .
$$

This formula is a generalization of (85).

Example 6.2 Set $I=(2), k=3$, and $n=4$. One has

$$
\beta_{(234)}=\beta_{(243)}+\beta_{(23)} \beta_{4}=\beta_{(252)}+\beta_{(24)} \beta_{3}+\beta_{(23)} \beta_{4}+\cdots .
$$

The last step of this process is

$$
\beta_{(234)}=\beta_{(23)} \beta_{4}+\beta_{(24)} \beta_{2}+\beta_{(25)} \beta_{2}+\beta_{(26)} \beta_{1}+\beta_{(27)} .
$$

After some iterations of (109), we obtain, for any composition $I>0$,

$$
\beta_{I}=\sum_{\phi \in \Phi_{I}} \beta^{\phi(I)} .
$$

Example 6.3 Set $I=(211)$. After two iterations of (109), we obtain

$$
\beta_{I}=\beta_{(22)}+\beta_{(21)} \beta_{1}=\beta_{4}+\beta_{3} \beta_{1}+\beta_{2} \beta_{2}+\beta_{3} \beta_{1}+\beta_{2} \beta_{1} \beta_{1} .
$$

Note that the $\beta_{n}$ are interpreted here as noncommutative variables. Note also that there are two occurrences of (31) in this expansion. Indeed, the flow $(1,1)$ maps $I$ to (310), while the flow $(1,0)$ maps it to $(301)$.

Flows can be linked with nondecreasing parking functions. Indeed, one can obtain from any flow $\phi \in \Phi_{I}$ a parking function $F \leq p(I)$ by applying the following construction from $p(I)$. The first $\phi_{s-1}$ parts equal to $s$ are replaced by parts equal to $s-1$, the first $\phi_{s-2}$ parts equal to $s-1$ are replaced by parts equal to $s-2$, and one continues in this way.

For example, for $I=(2222)$, one has

$$
p(I)=(11223344)
$$

The flow $\phi=(1,0,1)$ corresponds to the parking function (11123334), whose evaluation is $(3131)=\phi(I)$. Actually, this construction gives a bijection from $\Phi_{I}$ to the 
set of nondecreasing parking functions smaller than $p(I)$. This remark allows us to rewrite (112) as follows:

$$
\beta_{I}=\sum_{F \leq p(I)} \beta^{\mathrm{ev}(F)} .
$$

Note that different parking functions can have the same evaluation. That is why some compositions appear several times in this expansion. From $\beta_{n}=(-1)^{n}$, since all of the considered compositions have the same weight, we are now able to give the following theorem.

Theorem 6.4 The coefficient $\beta_{I}$ is given by

$$
\beta_{I}=(-1)^{|I|_{\sharp}} \sharp\{F \in \operatorname{NDPF}(|I|) / F \leq p(I)\} .
$$

From (32), (59), and this theorem we can deduce the following combinatorial identity.

Corollary 6.5 Let $F$ be a packed nondecreasing parking function, and let $s$ be the length of its evaluation. Then, the number $N$ of nondecreasing parking functions $G$ of the same size as $F$ and such that $G \leq F$ is

$$
N=\sum_{F \in \operatorname{NDPF}(s-1)} \prod_{k=1}^{s-1}\left(\begin{array}{c}
i_{s+1-k}+1 \\
m_{k}(F)
\end{array}\right) .
$$

\subsection{A combinatorial interpretation of the $\delta_{I}$}

In this section, we introduce a graphical representation for compositions. First, we represent any $I$ by a ribbon as in Sect. 2.1. Then, we label each box of the ribbon with the $k$ corresponding to the part $i_{k}$ of $I$ from which it comes. For example, (222) and (311) correspond respectively to

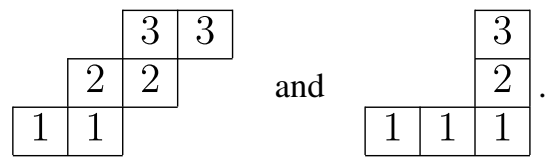

One can obtain $p(I)$ by reading the picture corresponding to $I$ from left to right and from the bottom to the top. For example, one has $p(222)=(112233)$ and $p(311)=$ (11123). Now, define $\delta_{I}^{\prime}$ as the number of the compositions $J$ for which the ribbon is at the top and to the left of the one corresponding to $I$ in the same rectangle, that is, $|I|=|J|, l(I)=l(J)$, and for all $1 \leq k \leq l(I)$,

$$
\sum_{s=1}^{k} j_{s} \leq \sum_{s=1}^{k} i_{s}
$$

Define $A_{I}$ as the set of these compositions. For example, for $I=(222)$, we will have 


$$
A_{I}=\{(213),(132),(123),(114),(222)\} .
$$

The elements of this set are given by the following figures:

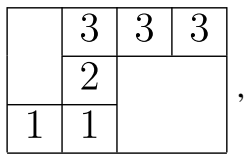

\begin{tabular}{|l|l|l|l|}
\hline \multicolumn{2}{|l|}{} & 3 & 3 \\
\hline 2 & 2 & 2 & \\
\cline { 1 - 2 } 1 & \multicolumn{2}{|c}{} \\
\cline { 1 - 2 }
\end{tabular}
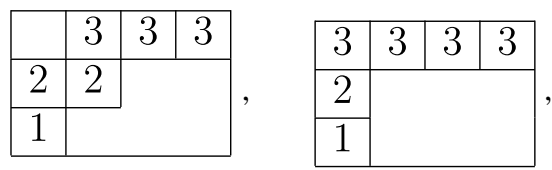

and

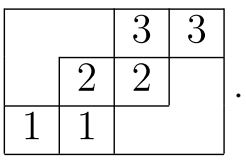

Namely, $A_{I}$ is the set of compositions $J$ of weight $|I|$ and of length $l(I)$ such that

$$
p(J) \geq p(I) .
$$

Actually, one also has

$$
\delta_{I}^{\prime}=(-1)^{|I|} \delta_{I}
$$

Indeed, one can see by studying the pictures that $(-1)^{|I|} \delta_{I}^{\prime}$ satisfies Propositions 5.1 and 5.2. Now, suppose that $i_{1}>1$ and define $J$ as the composition obtained by subtracting 1 from the first part of $I$. Then, the elements of $A_{J}$ can be obtained by removing the first cell of ribbons from $A_{I}$ starting from the left. On the other hand, denote by $K$ the composition obtained by adding the first two parts of $J$. Then, the elements of $A_{K}$ can be obtained by removing the first cell of ribbons from $A_{I}$ starting from the top. Hence,

$$
\delta_{I}^{\prime}=\delta_{J}^{\prime}+\delta_{K}^{\prime}
$$

From this deduce that $(-1)^{|I|} \delta_{I^{\prime}}$ satisfies Theorem 5.3, which gives (123).

Theorem 6.6 The coefficient $\delta_{I}$ is given for any I by

$$
\delta_{I}=(-1)^{n} \sharp\{J \vDash|I| / l(J)=l(I) \text { and } p(J) \geq p(I)\} .
$$

Note that from this one can explain the property $\delta_{I}=\delta_{\tilde{I}}$ by studying the ribbon pictures.

\section{Algebraic interpretations}

Let $I$ be a composition, and let $k$ and $n$ be positive integers. From Lemma 5.5 one has

$$
\beta_{I \cdot(k, n)}=\beta_{I \cdot(k+n)}-\beta_{I \cdot(k, n-1)} .
$$


Set $J=I^{\prime}$. From (59), one also has

$$
\begin{aligned}
(-1)^{k+n+|I|+l(I)} \alpha_{(n, k) \cdot J}= & (-1)^{k+n+|I|+l(I)+1} \alpha_{(n+k) \cdot J} \\
& +(-1)^{k+n+|I|+l(I)} \alpha_{(n-1, k) \cdot J},
\end{aligned}
$$

that is,

$$
\alpha_{(n, k) \cdot J}=\alpha_{(n-1, k) \cdot J}-\alpha_{(n+k) \cdot J} .
$$

From (126) and (128) we deduce the following proposition.

Proposition 7.1 Let I be a composition of a nonnegative integer, and let $k$ and $n$ be two positive integers. Then,

$$
\alpha_{(n, k) \cdot I}=\alpha_{(n+k+1) \cdot I}+\alpha_{(n+1, k) \cdot I}
$$

and

$$
\beta_{I \cdot(k, n)}=\beta_{I \cdot(k+n+1)}-\beta_{I \cdot(k, n+1)} .
$$

For any composition $I$, set $P_{I}=(-1)^{l(I)} R_{I}$. Now, consider two mutually commuting noncommutative alphabets $A$ and $B$, independent of each other. Denote by $f_{1}$, $f_{2}$, and $f_{3}$ the linear forms with values in the noncommutative symmetric functions of $A$, respectively defined by

$$
\begin{aligned}
f_{1}\left(\sum_{I} R_{I}(A) S^{I}(B)\right) & =\sigma_{1}^{\star}(B), \\
f_{2}\left(\sum_{I}(-1)^{l(I)} R_{I}(A) R_{I}(B)\right) & =\sigma_{1}^{\star}(B),
\end{aligned}
$$

and

$$
f_{3}\left(\sum_{I} R_{I}(A) \Lambda^{I}(B)\right)=\sigma_{1}^{\star}(B) .
$$

These definitions are equivalent to

$$
f_{1}\left(R_{I}(A)\right)=\alpha_{I}, \quad f_{2}\left(P_{I}(A)\right)=\beta_{I}, \quad \text { and } \quad f_{3}\left(R_{I}(A)\right)=\delta_{I} .
$$

From Theorem 5.3 we deduce that for all $n>1$ and for all compositions $I$,

$$
\begin{aligned}
f_{3}\left(R_{(n) \cdot I}(A)\right) & =\delta_{(n) \cdot I} \\
& =-\delta_{(n-1) \cdot I}-\delta_{\left(n-1+i_{1}\right) \cdot\left(i_{2}, i_{3}, \ldots\right)} \\
& =-f_{3}\left(R_{(n-1) \cdot I}(A)\right)-f_{3}\left(R_{\left(n-1+i_{1}\right) \cdot\left(i_{2}, i_{3}, \ldots\right)}(A)\right) .
\end{aligned}
$$

Hence,

$$
f_{3}\left(R_{(n) \cdot I}(A)\right)=-f_{3}\left(R_{(n-1) \cdot I}(A)\right)+R_{\left(n-1+i_{1}\right) \cdot\left(i_{2}, i_{3}, \ldots\right)}(A),
$$


that is,

$$
f_{3}\left(R_{(n) \cdot I}(A)\right)=-f_{3}\left(R_{n-1}(A) R_{I}(A)\right)
$$

(note that this identity is also true for $n=1$ from Proposition 5.2). An analogous process using Proposition 7.1 leads to

$$
f_{1}\left(R_{(n) \cdot I}(A)\right)=f_{1}\left(R_{n+1}(A) R_{I}(A)\right)
$$

Now,

$$
P_{I} P_{n+1}=(-1)^{l(I)+1}\left(R_{I \cdot(n+1)}+R_{\left(i_{1}, \ldots, i_{s-1}, i_{s}+n+1\right)}\right),
$$

where $s=l(I)$. Hence,

$$
P_{I} P_{n+1}=P_{I \cdot(n+1)}-P_{\left(i_{1}, \ldots, i_{s-1}, i_{s}+n+1\right)} .
$$

Then, formula (130) gives a functional equation of which $f_{2}$ is a solution. Summarizing, we then have the following:

Proposition 7.2 The linear forms $f_{1}, f_{2}$, and $f_{3}$ are respectively solutions of the functional equations

$$
\begin{aligned}
& f_{1}\left(R_{(n) \cdot I}(A)\right)=f_{1}\left(R_{n+1}(A) R_{I}(A)\right), \\
& f_{2}\left(P_{I \cdot(n)}(A)\right)=-f_{2}\left(P_{I}(A) P_{n+1}(A)\right),
\end{aligned}
$$

and

$$
f_{3}\left(R_{(n) \cdot I}(A)\right)=-f_{3}\left(R_{n-1}(A) R_{I}(A)\right)
$$

\section{Links between the $\alpha_{I}$ and the $\delta_{I}$}

From (35) and (45) one can deduce that for all compositions $I$,

$$
\delta_{I}=(-1)^{|I|} \sum_{F \in \operatorname{NDPF}(l(I)-1)} \prod_{k=1}^{l(I)-1}\left(\begin{array}{l}
i_{k} \\
a_{k}
\end{array}\right) .
$$

If we compare this formula with (28), we can see that the $\alpha_{I}$ and the $\delta_{I}$ are linked by

$$
\alpha_{I}=(-1)^{|I|+l(I)-l(I)} \delta_{\left(i_{1}+1, i_{2}+1, \ldots\right)}=(-1)^{|I|} \delta_{\left(i_{1}+1, i_{2}+1, \ldots\right)} .
$$

Remark that by using this formula, one can deduce (140) from (142) .

Now, let us consider the algebra morphism $\phi: \mathbf{S y m} \mapsto \mathbf{S y m}$ defined by $\phi\left(\Lambda_{1}\right)=0$ and $\phi\left(\Lambda_{n}\right)=(-1)^{n-1} S_{n-1}$ for $n \geq 1$. Then,

$$
\phi\left(\sigma_{1}^{\star}\right)=\phi\left(\sum_{I} \delta_{I} \Lambda^{I}\right)=\sum_{I}(-1)^{|I|} \delta_{\left(i_{1}+1, i_{2}+1, \ldots\right)} S^{I}
$$


Hence,

$$
\phi\left(\sigma_{1}^{\star}\right)=\sum_{I} \alpha_{I} S^{I}=\sigma_{1}^{\star},
$$

so that $\sigma_{1}^{\star}$ is invariant under this morphism.

By turning the pictures of Sect. 6.2 upside down, one can say from Theorem 6.6 that $\left|\delta_{I}\right|$ is the number of packed nondecreasing parking functions $F$ with the same last part as $p\left(I^{\prime}\right)$ and such that $F \leq p\left(I^{\prime}\right)$. Hence, the identity

$$
\left|\alpha_{I}\right|=\left|\beta_{I^{\prime}}\right|=\left|\delta_{\left(i_{1}+1, i_{2}+1, \ldots\right)}\right|,
$$

obtained from (144) and (59), gives, together with (6.6), the following combinatorial identity.

Proposition 8.1 Let $F$ be a packed nondecreasing parking function of size $n$ with last part $s$. Denote by $\tilde{F}$ the parking function obtained by adding to $F$ a part of each value from 1 to $s$ (for example, if $F=(1123)$, then $\tilde{F}=(1112233)$ ).

The number of the packed nondecreasing parking functions $G$ of the same size and the same last term as $\tilde{F}$ and such that $G \leq \tilde{F}$ is then equal to the number of all the nondecreasing parking functions $G$ of size $n$ such that $G \leq F$.

We shall now give an explicit bijection. To this end, we shall need a second graphical representation of nondecreasing parking functions. This time, we consider ribbons whose last column at the right has only one cell. We assume that the first cell of any ribbon has height 1 , and that each ribbon corresponds to the sequence obtained by reading the height of the first cell at the bottom of each column. We start this process with the second column from the left. For example, $(1,1,3,3,4)$ corresponds to

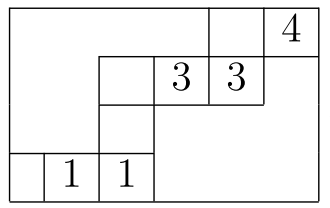

This graphical representation will be called the second correspondence, and the graphical representation for nondecreasing parking functions introduced in Sect. 6.2 will be called the first correspondence.

Given a nondecreasing parking function $F$, we can see that the set of nondecreasing parking functions smaller than $F$ and of the same size coincides with the set of all the ribbons at the bottom and at the right of the ribbon corresponding to $F$ and of the same length.

Now, suppose that $F$ is a packed nondecreasing parking function, and let $\tilde{F}$ be the parking function obtained from $F$ as in Proposition 8.1. Then, the ribbon corresponding to $F$ under the first correspondence is the same as the one corresponding to $\tilde{F}$ under the second one.

Finally, let $G$ be a nondecreasing parking function of the same size as $F$ and such that $G \leq F$. By adding cells at the top of the last cell of the ribbon corresponding to $G$ 
under the second correspondence, one can obtain a new ribbon with the same height as the one corresponding to $F$. Under the first correspondence, this ribbon coincides with a packed nondecreasing parking function $\tilde{G}$ with the same size, the same last term as $\tilde{F}$, and such that $\tilde{G} \leq \tilde{F}$. For example, the inequality $(1133) \leq(1234)$, which is true since the ribbon

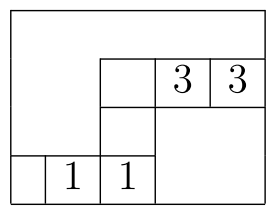

is at the bottom and at the right of

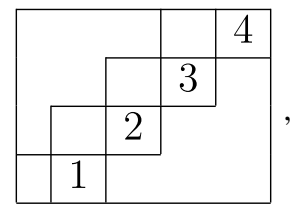

leads to $(11123334) \leq(11223344)$, as we can see in the following picture:

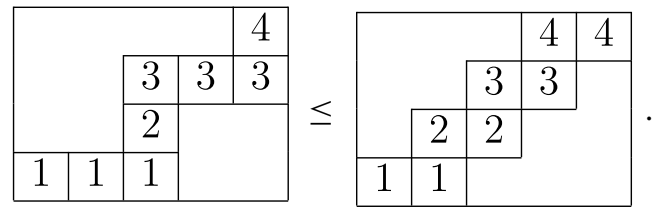

Let $F$ be a packed nondecreasing parking function of size $n$. Let $\tilde{F}$ be the parking function obtained from $F$ as in Proposition 8.1, and $p$ its size. Define $A_{F}$ as the set of the $G \in \operatorname{NDPF}(n)$ such that $G \leq F$, and define $B_{F}$ as the set of the $G \in \operatorname{NDPF}(p)$ packed, with the same last term as $\tilde{F}$ and such that $G \leq \tilde{F}$. Then, we obtain a bijection

$$
f: A_{F} \rightarrow B_{F}
$$

by setting that for all $G \in A_{F}, f(G)$ is obtained by adding one part $s$ to $G$ for each $s$ from 1 to the last term of $\tilde{F}$.

For example, for $F=(12334)$ and $G=(11333) \in A_{F}$, we obtain $\tilde{F}=$ (112233344) and $f(G)=(111233334) \in B_{F}$.

\section{A one-parameter deformation of $\mathcal{H}$}

In this section, we will be interested in a deformation $\mathcal{H}_{\gamma}$ of the algebra $\mathcal{H}$ of noncommutative formal diffeomorphisms, where $\gamma$ is a real parameter. As an associative algebra, $\mathcal{H}_{\gamma}$ coincides with the algebra of noncommutative symmetric functions. 
Its coproduct $\Delta_{\gamma}$ is given on complete symmetric functions by the formula

$$
\Delta_{\gamma} S_{n}(A)=\sum_{k=0}^{n} S_{k}(A) \otimes S_{n-k}((k \gamma+1) A) .
$$

This deformation of the noncommutative Faà di Bruno Hopf algebra of [1] has been recently discovered by Foissy [3] in his investigation of combinatorial DysonSchwinger equations in the Connes-Kreimer algebra. As a Hopf algebra, $\mathcal{H}_{0}$ is the algebra of noncommutative symmetric functions, and the noncommutative Faà di Bruno Hopf algebra is the case $\gamma=1$. Foissy [3] shows that for $\gamma \neq 0, \mathcal{H}_{\gamma}$ is isomorphic to $\mathcal{H}_{1}=\mathcal{H}$. This isomorphism can be compactly described as follows.

\section{Theorem 9.1}

$$
\begin{aligned}
\mathcal{H}_{\gamma} & \rightarrow \mathcal{H}_{1} \\
F(A) & \mapsto F\left(\frac{1}{\gamma} A\right)
\end{aligned}
$$

is an isomorphism of Hopf algebras.

Proof Denoting by $\bar{\Delta}_{\gamma}$ the coproduct which maps each $F$ to the image of $\Delta_{\gamma}(F)$ under the isomorphism

$$
G \otimes H \mapsto H \otimes G
$$

one has

$$
\begin{aligned}
\Delta_{1}\left(\sigma_{1}\right) & =\sum_{n} \sum_{k=0}^{n} S^{k} \otimes S^{n-k}((k+1) A) \\
& =\sum_{k \geq 0} \sum_{m \geq 0} S^{k} \otimes S^{m}((k+1) A) \\
& =\sum_{k \geq 0} S^{k} \otimes \sigma_{1}((k+1) A),
\end{aligned}
$$

so that

$$
\bar{\Delta}_{1}\left(\Phi\left(\sigma_{1}\right)\right)=\left(\bar{\Delta}_{1}\left(\sigma_{1}\right)\right)^{\frac{1}{\gamma}}=\left(\sum_{k \geq 0} \sigma_{1}^{k+1} \otimes S^{k}\right)^{\frac{1}{\gamma}}
$$

Hence,

$$
\bar{\Delta}_{1}\left(\Phi\left(\sigma_{1}\right)\right)=\left(\sigma_{1} \otimes 1\right)^{\frac{1}{\gamma}}\left(\sum_{k \geq 0} \sigma_{1}^{k} \otimes S^{k}\right)^{\frac{1}{\gamma}} .
$$

On the other hand, one has

$$
\sigma_{\sigma_{1}(A)}(B)=\sum_{k \geq 0} \sigma_{1}(A)^{k} S^{k}(B) .
$$


By means of the identification

$$
F \otimes G=F(A) G(B),
$$

we then obtain

$$
\left(\sum_{k \geq 0} \sigma_{1}^{k} \otimes S^{k}\right)^{\frac{1}{\gamma}}=\sigma_{\sigma_{1}(A)}\left(\frac{1}{\gamma} B\right)=\sum_{k \geq 0} \sigma_{1}^{k} \otimes S^{k}\left(\frac{1}{\gamma} A\right),
$$

so that

$$
\begin{aligned}
\bar{\Delta}_{1}(\Phi(\sigma)) & =(\sigma \otimes 1)^{\frac{1}{\gamma}} \sum_{k \geq 0} \sigma_{1}^{k} \otimes S^{k}\left(\frac{1}{\gamma} A\right) \\
& =\left(\sigma_{1}\left(\frac{1}{\gamma} A\right) \otimes 1\right) \sum_{k \geq 0} \sigma\left(\frac{1}{\gamma} A\right)^{k \gamma} \otimes S^{k}\left(\frac{1}{\gamma} A\right) .
\end{aligned}
$$

Finally, we then have

$$
\Delta_{1}\left(\Phi\left(\sigma_{1}\right)\right)=\sum_{k \geq 0} S^{k}\left(\frac{1}{\gamma} A\right) \otimes \sigma_{1}\left(\frac{1}{\gamma} A\right)^{k \gamma+1}=\Phi\left(\Delta_{\gamma}\left(\sigma_{1}\right)\right) .
$$

Denote by $s_{\gamma}$ the antipode of $\mathcal{H}_{\gamma}$, and define respectively by $\tilde{\alpha}_{I}$ and $\tilde{\delta}_{I}$ the coefficients in the following expansions:

$$
s_{\gamma}\left(\sigma_{1}\right)=\sum_{I} \tilde{\alpha}_{I} S^{I}=\sum_{I} \tilde{\delta}_{I} \Lambda^{I} .
$$

In [2], we give an explicit formula for the antipode $s_{\gamma}$ that is a natural deformation of the Lagrange inversion formula of Brouder-Frabetti-Krattenthaler. Namely, we determine explicitly the coefficients $\tilde{\alpha}_{I}$ by

$$
\tilde{\alpha}_{I}=(-1)^{l(I)} \sum_{F \in \operatorname{NDPF}(l(I)-1)} \prod_{k=1}^{l(I)-1}\left(\begin{array}{c}
i_{k} \gamma+1 \\
m_{k}(F)
\end{array}\right) .
$$

As we can see, formula (28) is recovered in the case $\gamma=1$.

\section{An algebra morphism}

In this section, we suppose that $\gamma$ is a positive integer, and we introduce an algebra morphism $\phi_{\gamma}$ such that

$$
\phi_{\gamma} \circ s=s_{\gamma}
$$

We look at some properties of this morphism, and we determine explicitly its action on various bases. 
10.1 Definition of the morphism $\phi_{\gamma}$ and links with $\mathcal{H}_{\gamma}$

Let $\gamma$ be a positive integer, and let $\phi_{\gamma}$ be the algebra morphism defined from Sym to itself by

$$
\phi_{\gamma}\left(S_{n}\right)= \begin{cases}S_{n / \gamma} & \text { if } \gamma \mid n, \\ 0 & \text { otherwise. }\end{cases}
$$

It is easy to see that the $\phi_{\gamma}$ satisfy

$$
\phi_{\gamma} \circ \phi_{\gamma^{\prime}}=\phi_{\gamma \gamma^{\prime}}
$$

We define the operator $\psi_{\gamma}$ as the adjoint of $\phi_{\gamma}$, in the sense that

$$
\left\langle\phi_{\gamma}(F), G\right\rangle=\left\langle F, \psi_{\gamma}(G)\right\rangle \text {. }
$$

Here, $F$ is a noncommutative symmetric function, $G$ is a quasi-symmetric function, and the bracket corresponds to the duality between $\mathcal{H}_{0}$ and the classical Hopf algebra of quasi-symmetric functions. It is easy to see that $\psi_{\gamma}$ is defined from QSym to itself by

$$
\psi_{\gamma}\left(M_{I}\right)=M_{\left(\gamma i_{1}, \gamma i_{2}, \ldots\right)},
$$

where $\left(M_{I}\right)$ is the basis of monomial quasi-symmetric functions. This operator acts on any quasi-symmetric function $F$ by replacing all the monomials by their $\gamma$ th power in the polynomial realization of $F$. Hence, it is also an algebra morphism. We can also deduce from $\sigma_{1}^{*}=\sum_{I} \alpha_{I} S^{I}$ and the definition of $\phi_{\gamma}$ that

$$
\phi_{\gamma}\left(\sigma_{1}^{*}\right)=\sum_{I} \tilde{\alpha}_{\left(\gamma i_{1}, \gamma i_{2}, \ldots\right)} S^{I}
$$

On the other hand, we deduce from (165) that

$$
\tilde{\alpha}_{I}=\alpha_{\left(\gamma i_{1}, \gamma i_{2}, \ldots\right)}
$$

From (59) and (116) we have a combinatorial interpretation for $\alpha_{I}$, that is,

$$
\alpha_{I}=(-1)^{l(I)} \sharp\left\{F \in \operatorname{NDPF}(|I|) / F \leq p\left(I^{\prime}\right)\right\} .
$$

From (172) we deduce the following combinatorial interpretation for $\tilde{\alpha}_{I}$.

Proposition 10.1 When $\gamma$ is a positive integer, the corresponding coefficient $\tilde{\alpha}_{I}$ is given for any composition I by

$$
\tilde{\alpha}_{I}=(-1)^{l(I)} \sharp\left\{F \in \operatorname{NDPF}(\gamma|I|) / F \leq p\left(I^{\prime}\right)^{\gamma}\right\},
$$

where for any nondecreasing parking function $F, F^{\gamma}$ is the parking function obtained by concatenating $\gamma$ parking functions equal to $F$ and by reordering the obtained sequence. For example, $(1123)^{3}=(111111222333)$.

Now, we can deduce from (171) the following proposition. 
Proposition 10.2 The antipode $s$ of $\mathcal{H}$ and the antipode $s_{\gamma}$ of $\mathcal{H}_{\gamma}$ are linked to each other by the formula

$$
s_{\gamma}=\phi_{\gamma} \circ s
$$

Now, let

$$
\Psi_{\gamma}: \mathbf{S y m} \mapsto \mathbf{S y m}
$$

be the algebra isomorphism $F(A) \mapsto F(\gamma A)$. The compositional inverse of this isomorphism maps $F(A)$ to $F\left(\frac{1}{\gamma} A\right)$. From Proposition 9.1 we have

$$
s \circ \Psi_{\gamma}^{-1}=\Psi_{\gamma}^{-1} \circ s_{\gamma},
$$

that is,

$$
s=\Psi_{\gamma}^{-1} \circ s_{\gamma} \circ \Psi_{\gamma} .
$$

From (178) and (175) we deduce the following proposition.

Proposition 10.3 The antipode $s$ of $\mathcal{H}_{1}$ is a solution of an infinity of functional equations, of the general form

$$
s=\Psi_{\gamma}^{-1} \circ \phi_{\gamma} \circ s \circ \Psi_{\gamma}
$$

where $\gamma$ is a positive integer.

10.2 Action of $\phi_{\gamma}$ on the $\Lambda^{I}$

For any homogeneous noncommutative symmetric function $F$ such that its degree is not a multiple of $\gamma$, one has, by the definition of $\phi_{\gamma}$,

$$
\phi_{\gamma}(F)=0 \text {. }
$$

Now, let $n$ be a multiple of $\gamma$, and let us set $k=n / \gamma$. We then have

$$
\Lambda_{n}=(-1)^{n} \sum_{I \models n}(-1)^{l(I)} S^{I} .
$$

In this equation, the $S^{I}$ which can give a contribution to $\phi_{\gamma}\left(\Lambda_{n}\right)$ correspond to the $I$ for which all the parts are multiples of $\gamma$. These $I$ are exactly the $\left(\gamma j_{1}, \gamma j_{2}, \ldots\right)$ where $J \vDash k$, and for these compositions, one has

$$
\phi_{\gamma}\left(S^{I}\right)=S^{J} .
$$

Hence,

$$
\phi_{\gamma}\left(\Lambda_{n}\right)=(-1)^{n} \sum_{J \models k}(-1)^{l(J)} S^{J}=(-1)^{n-k} \Lambda_{k},
$$

and from this we deduce the following proposition. 
Proposition 10.4 Let $n$ be a positive integer. Then, the action of $\phi_{\gamma}$ on $\Lambda_{n}$ is given by

$$
\phi_{\gamma}\left(\Lambda_{n}\right)= \begin{cases}(-1)^{n-\frac{n}{\gamma}} \Lambda_{n / \gamma} & \text { if } \gamma \mid n \\ 0 & \text { otherwise. }\end{cases}
$$

Note that since $\phi_{\gamma}$ is an algebra morphism, one can deduce from that its action on the $\Lambda^{I}$.

\subsection{Action of $\phi_{\gamma}$ on the $R_{I}$}

Let $I$ be a composition whose weight $n$ is a multiple of $\gamma$, and let us set $k=\frac{n}{\gamma}$. We then have

$$
R_{I}=\sum_{J \geq I}(-1)^{l(I)-l(J)} S^{J}
$$

An $S^{J}$ can give a contribution to $\phi_{\gamma}\left(R_{I}\right)$ only if all the parts of $J$ are multiples of $\gamma$, it follows that $J \geq K$, where $K$ is the composition with $k$ parts all equal to $\gamma$. Hence,

$$
\phi_{\gamma}\left(R_{I}\right)=\sum_{J \geq I, J \geq K}(-1)^{l(I)-l(J)} \phi_{\gamma}\left(S^{J}\right) .
$$

Let $L$ be the smallest common reverse refinement of $I$ and $K$. Then, we can rewrite this equality as

$$
\phi_{\gamma}\left(R_{I}\right)=\sum_{J \geq L}(-1)^{l(I)-l(J)} \phi_{\gamma}\left(S^{J}\right) .
$$

Let $\hat{L}$ be the composition obtained by dividing all the parts of $L$ by $\gamma$. By the definition of $\phi_{\gamma}$, we then obtain

$$
\phi_{\gamma}\left(R_{I}\right)=\sum_{J \geq \hat{L}}(-1)^{l(I)-l(J)} S^{J}=(-1)^{l(I)-l(L)} R_{L} .
$$

From this we can deduce the following proposition.

Proposition 10.5 Let I be a composition. If $|I|$ is not a multiple of $\gamma$, then

$$
\phi_{\gamma}\left(R_{I}\right)=0 .
$$

If $|I|$ is a multiple of $\gamma$, then

$$
\phi_{\gamma}\left(R_{I}\right)=(-1)^{l(I)-l(J)} R_{J},
$$

where $J$ is the composition obtained by dividing by $\gamma$ all the parts of the smallest common reverse refinement of I and the composition with weight $|I|$ all of whose parts are equal to $\gamma$. 


\section{A new closed formula for the antipode of $\mathcal{H}_{\gamma}$}

From Proposition 10.4 we have

$$
\phi_{\gamma}\left(\sigma_{1}^{\star}\right)=\sum_{I} \delta_{I} \phi_{\gamma}\left(\Lambda^{I}\right)=\sum_{I}(-1)^{|I|(\gamma-1)} \delta_{\left(i_{1} \gamma, i_{2} \gamma, \ldots\right)} \Lambda^{I}
$$

Since

$$
\phi_{\gamma}\left(\sigma_{1}^{\star}\right)=\sum_{I} \tilde{\delta}_{I} \Lambda^{I}
$$

one can deduce that

$$
\tilde{\delta}_{I}=(-1)^{|I|(\gamma-1)} \delta_{\left(i_{1} \gamma, i_{2} \gamma, \ldots\right)} .
$$

Since the sign of $\delta_{J}$ is $(-1)^{|J|}$ for all compositions $J(125)$, the sign of $\tilde{\delta}_{I}$ is then

$$
(-1)^{|I|(\gamma-1)+|I| \gamma}=(-1)^{|I|} .
$$

On the other hand, one has

$$
\left|\tilde{\delta}_{I}\right|=\left|\delta_{\left(i_{1} \gamma, i_{2} \gamma, \ldots\right)}\right| .
$$

Then, one can give a closed formula for $\tilde{\delta}_{I}$ when $\gamma$ is a positive integer. Actually, this condition is not necessary, since $\tilde{\delta}_{I}$ is a polynomial in $\gamma$.

Proposition 11.1 The value of $\tilde{\delta}_{I}$ is given for $\gamma \in \mathbb{R}^{*}$ by

$$
\tilde{\delta}_{I}=(-1)^{|I|} \sum_{F \in \operatorname{NDPF}(l(I)-1)} \prod_{k}\left(\begin{array}{c}
i_{k} \gamma \\
m_{k}(F)
\end{array}\right) .
$$

Note that $\left|\tilde{\delta}_{I}\right|$ and $\tilde{\alpha}_{I}$ do not depend on the last part of $I$.

When $\gamma$ is a positive integer, we deduce from (195) and (125) the following combinatorial interpretation for $\tilde{\delta}_{I}$.

Proposition 11.2 When $\gamma$ is a positive integer, the corresponding coefficient $\tilde{\delta}_{I}$ is given for any composition I by

$$
\left.\tilde{\delta}_{I}=(-1)^{|I|_{\sharp}} \sharp J \models \gamma|I| / l(J)=l(I) \text { and } p(J) \geq p(I)^{\gamma}\right\},
$$

where for any nondecreasing parking function $F, F^{\gamma}$ is the parking function obtained by concatenating $\gamma$ parking functions equal to $F$ and by reordering the obtained sequence.

\section{References}

1. Brouder, C., Frabetti, A., Krattenthaler, C.: Non-commutative Hopf algebra of formal diffeomorphisms (2004). math.QA/0406117 
2. Bultel, J.P.: A one-parameter deformation of the noncommutative Lagrange inversion formula. Int. J. Algebra Comput. doi:10.1142/S0218196711006662

3. Foissy, L.: Faà di Bruno subalgebras of the Hopf algebra of planar trees from combinatorial DysonSchwinger equations. Adv. Math. 218(1), 136-162 (2008)

4. Gelfand, I.M., Krob, D., Lascoux, A., Leclerc, B., Retakh, V.S., Thibon, J.-Y.: Noncommutative symmetric functions. Adv. Math. 112, 218-348 (1995)

5. Gessel, I.: Noncommutative generalization and $q$-analog of the Lagrange inversion formula. Trans. Am. Math. Soc. 257(2), 455-482 (1980)

6. Krob, D., Leclerc, B., Thibon, J.-Y.: Noncommutative symmetric functions II: transformations of alphabets. Int. J. Algebra Comput. 7, 181-264 (1997)

7. Lascoux, A.: Symmetric Functions and Combinatorial Operators on Polynomials. CBMS Regional Conference Series in Mathematics, vol. 99. Am. Math. Soc., Providence (2003). xii+268 pp.

8. Lenart, C.: Lagrange inversion and Schur functions. J. Algebr. Comb. 11(1), 69-78 (2000)

9. Macdonald, I.G.: Symmetric Functions and Hall Polynomials, 2nd edn. Oxford University Press, London (1995)

10. Novelli, J.-C., Thibon, J.-Y.: Noncommutative symmetric functions and Lagrange inversion. Adv. Appl. Math. 40, 8-35 (2008)

11. Pak, I., Postnikov, A., Retakh, V.S.: Noncommutative Lagrange theorem and inversion polynomials, preprint (1995). Available at http://www-math.mit.edu/ pak/research.html 